\title{
EDQM-HC Administration Method Terminology
}

National Cancer Institute

\section{Source}

National Cancer Institute. EDQM-HC Administration Method Terminology. NCI

Thesaurus. Code C150112.

Terminology subset about general methods by which a pharmaceutical product is intended to be administered to the patient. 